\title{
Der konsortiale Bestandsaufbau als kooperative Informationsinfrastruktur. Die AG Erwerbungskoordinierung der sächsischen Hochschulbibliotheken
}

Erwerbungskoordinierung bedeutet weit mehr als bisweilen unterstellt, wenn sie manchmal als Einkaufsgemeinschaft bezeichnet wird. Tatsächlich war zunächst ihr hauptsächlicher Zweck, vor allem Preisvorteile zu erzielen, indem sich mehrere Bibliotheken zusammenschließen und gemeinsam Produkte lizenzieren oder kaufen. Erwerbungskonsortien haben sich jedoch in den vergangenen Jahren weiterentwickelt, denn immer öfter geht es auch um technische Standards oder um einheitliche Services. Begründet wurde die Arbeitsgruppe Erwerbungskoordinierung in Sachsen 1994. ${ }^{1}$ Elektronische Ressourcen, denen zudem wenig Etat zugewiesen war, spielten damals noch eine Nebenrolle. Dies änderte sich sowohl durch die allgemeine Entwicklung hin zum Digitalen als auch aufgrund der hohen Preissteigerung bei wissenschaftlichen Journalen, die unter dem Begriff „Zeitschriftenkrise“ firmiert. Die zunehmende Lizenzierung elektronischer Produkte sollte diesen Trend aufhalten und gleichzeitig die abgestimmte konsortiale Erwerbung Kräfte und Mittel bündeln. Letzteres ist gelungen, ersteres nicht, wenngleich große Preissteigerungen nicht mehr wie früher durchgesetzt werden können. Das Bibliothekssystem Sachsen (2008) ging auf die konsortiale Erwerbung ein, die auch bei der Monografienanschaffung Wirkung zeigen sollte. ${ }^{2}$ Dies ist zwar nicht wie geplant realisiert worden, jedoch

1 Vgl. Golsch, Michael u. Jana Herrmann: Erwerbungskoordinierung in Sachsen. Eine Zwischenbilanz. In: BIS - Das Magazin der Bibliotheken in Sachsen 8 (2015) H. 2. S. 82-85. http://slub.qucosa.de/api/qucosa\%3A5552/attachment/ATT-0/. Einen Überblick zur Erwerbskoordination von Konsortien für Hochschulen verschafft: Golsch, Michael: Koordiniert lizenzieren. Der Beitrag der Bibliothekskonsortien zur Informationsversorgung der Hochschulen. In: Handbuch Hochschulbibliothekssysteme. Leistungsfähige Informationsinfrastrukturen für Wissenschaft und Studium. Hrsg. von Konstanze Söllner u. Wilfried Sühl-Strohmenger. Berlin, Boston: de Gruyter 2014. S. 383-398. Alle aufgeführten Internetressourcen wurden zuletzt am 7. Dezember 2017 aufgerufen.

2 Vgl. Sächsisches Staatsministerium für Wissenschaft und Kunst (Hrsg.): Bibliothekssystem Sachsen. Struktur- und Entwicklungsplan für die wissenschaftliche Literatur- und Informationsversorgung im Freistaat Sachsen. Bearbeitet von Achim Bonte u. Joachim Linek. Dresden: Sächsisches Staatsministerium für Wissenschaft und Kunst 2008, S. 45-49. http://www.qucosa.de/fileadmin/data/qucosa/documents/169/1233064882461-0127.pdf.

ə Open Access. (c) 2018 Konstantin Hermann, publiziert von De Gruyter. (c) BY-Nc-ND Dieses Werk ist lizenziert unter der Creative Commons Attribution-NonCommercial-NoDerivatives 4.0 Lizenz. 
konnten die Ideen, die aus der Konzeption des Bibliothekssystems Sachsen für die Lizenzierung elektronischer Ressourcen erwuchsen, umgesetzt werden. So wurde im Doppelhaushalt 2009/2010 eine eigene Titelgruppe für die konsortiale Erwerbung verankert, die mit 1,5 Millionen Euro ausgestattet war. Die AG erhielt in dieser Zeit zudem eine eigene Webseite und wurde damit öffentlich sichtbar. ${ }^{3}$

Im Folgenden wird auf das Modell der AG Erwerbungskoordinierung eingegangen, dabei weniger die Geschichte betrachtet, ${ }^{4}$ vielmehr der Schwerpunkt auf die aktuelle Entwicklung seit 2015 gelegt. Besonders richtet sich der Blick auf die Zukunft dieser Art der informationsinfrastrukturellen Zusammenarbeit, die aktuell vor großen Herausforderungen steht - nicht zuletzt, weil das Lizenzierungsgeschäft stets Termingeschäft ist.

\section{Die AG Erwerbungskoordinierung der sächsischen Hochschulbibliotheken}

Die AG Erwerbungskoordinierung ist ein Zusammenschluss fast aller Hochschulbibliotheken in Trägerschaft des Freistaats Sachsen, die auch in der GASCO, dem Zusammenschluss der Konsortien der deutschsprachigen Länder, mitwirkt. 2017 trat noch die Bibliothek der Palucca Hochschule für Tanz Dresden bei, sodass die Universitäts-, die Hochschulbibliotheken sowie viele der Kunst- und Musikhochschulbibliotheken, die Bibliotheken der Berufsakademie Sachsen und die Bibliothek der Evangelischen Hochschule Dresden Mitglieder des Konsortiums sind. Aus der Bandbreite der gelehrten Fächer an den jeweiligen Hochschulen ergibt sich die Lizenzierung vor allem großer, fachlich übergreifender Pakete, die den Bedarf aller oder zumindest der meisten Einrichtungen decken.

Bewusst wurde die AG nicht in einen eingetragenen Verein oder eine sonstige fixierte Organisationsform überführt. Die rechtlich lose Verbindung spiegelt jedoch nicht die intensive Zusammenarbeit der Partner wider, die sich dreioder viermal im Jahr zu Konsortialsitzungen treffen, die jedes Mal in einer ande-

3 Heute: https://www.bibag-sachsen.de/ag-erwerbungskoordinierung-konsortialvertraege/ startseite/.

$4 \mathrm{Zu}$ den vergangenen Entwicklungen bleibt auf die beiden einschlägigen Beiträge in $B I S$ Das Magazin der Bibliotheken in Sachsen zu verweisen: Golsch/Herrmann, Erwerbungskoordinierung (wie Anm. 1); Golsch, Michael: Landeslizenzen für die Wissenschaft. Sachsens Konsortialstrategie ist erfolgreich. In: BIS - Das Magazin der Bibliotheken in Sachsen 9 (2016) H. 2. S. 83-85. http://slub.qucosa.de/api/qucosa\%3A7610/attachment/ATT-0/. In den Geschäftsberichten der SLUB bildet das Konsortium keine eigene Komponente. 
ren Teilnehmerbibliothek in Sachsen stattfinden. Dies bietet die Gelegenheit, die Gegebenheiten und Veränderungen der Einrichtungen vor Ort kennenzulernen und symbolisiert zugleich den kooperativen Ansatz. Vertreten werden die Bibliotheken durch die entsprechenden Erwerbungsleiter, die E-RessourcenManager oder bei kleineren Einrichtungen durch die Leiter der Bibliothek selbst. Seit 2009 liegt die Konsortialführung gemäß der gesetzlich zugewiesenen Verantwortung als Staatsbibliothek und Koordinierungsinstanz des Freistaats für das wissenschaftliche Bibliothekswesen bei der SLUB Dresden, in persona beim Abteilungsleiter Bestandsentwicklung und Metadaten. Als stellvertretende Leiterin agiert die Bereichsleiterin Medienbearbeitung der UB Chemnitz. Um die Zusammenarbeit nachhaltig $\mathrm{zu}$ sichern und transparent $\mathrm{zu}$ gestalten, werden relevante Informationen aus dem Konsortium im SLUB-Intranet in einem eigenen Bereich abgelegt, in den etwa Verträge oder Mittelübersichten eingestellt werden und in dem kollaborativ an Dokumenten gearbeitet wird. Die Bibliotheken erhalten dafür eigene Zugänge in das Intranet. Ebenso dient die Dokumentation auf den internen Webseiten der Kontrolle des Mittelabflusses der AG Erwerbungskoordinierung. ${ }^{5}$

Die Verhandlung mit den Anbietern erfolgt dezentral, wobei sich die SLUB als Konsortialführerin jedoch an allen Gesprächen frühzeitig beteiligt. ${ }^{6}$ Alle Konditionen werden in den Konsortialsitzungen beraten und entschieden. Besonders die Universitätsbibliotheken verhandeln meist die großen Verträge, während die Hochschulbibliotheken oft Lizenzen mit geringerem finanziellen Umfang aushandeln. Bisweilen resultieren daraus mehrmonatige Gespräche, die in den meisten Fällen in einen erfolgreichen Vertragsabschluss münden. Innerhalb des Konsortiums gelang es bereits, Produkte, die bisher keinen konsortialen Zugang zuließen, den Teilnehmerbibliotheken zur Verfügung zu stellen. Genauso gehörten und gehören aber auch Ablehnungen von Angeboten größerer Verlage zum Geschäft, wenn diese Preis- und Servicevorstellungen haben, die für das Konsortium inakzeptabel sind, und wenn außerdem die weiteren Verhandlungen keine wesentlichen Fortschritte erzielen. Genau wie im Herbst 2015 die konsortial gehaltenen Zeitschriften eines Anbieters gekündigt wurden, ${ }^{7}$ wurde zwei Jahre später, im Herbst 2017, eine andere große Konsortiallizenz

5 Konsortialmittel können nicht in das nächste Jahr übertragen werden. Die Bibliotheken sind daher an die Mittelverwendung im aktuellen Haushaltsjahr gebunden.

6 Weiterhin ist die SLUB für die sächsischen und thüringischen teilnehmenden Bibliotheken Träger der Infrastruktur im „Beuth-Konsortium“, das den Onlinezugang zur Perinorm-Datenbank gewährleistet. Gehostet wird dieses Angebot in DBoD (Databases on Demand) der SLUB. Die Normen sind für die Discovery-Kataloge tiefenerschlossen und erlauben somit eine wesentlich bessere Recherche und Nutzung.

7 Allerdings wurde nach der Einigung ein Dreijahresvertrag geschlossen. 
nicht weitergeführt, da die Preisvorstellungen und -berechnungsgrundlagen aus Sicht aller Konsortialteilnehmer nicht annehmbar waren.

\section{Die Lizenzierung elektronischer Medien im Lan- desdigitalisierungsprogramm für Wissenschaft und Kultur}

Mit dem Beginn des „Landesdigitalisierungsprogrammes für Wissenschaft und Kultur des Freistaates Sachsen“ (LDP) erfuhr die konsortiale Erwerbungsstrategie der sächsischen Hochschulbibliotheken eine wichtige Unterstützung und Bestätigung. Das LDP, in seinem inhaltlichen Zuschnitt in Deutschland gleichermaßen einzigartig und anerkannt, besteht aus drei sich ergänzenden Komponenten: Massen- und Einzelstückdigitalisierung für Universitätsbibliotheken und andere wissenschaftliche Einrichtungen, vor allem Bibliotheken, die Langzeitarchivierung der daraus entstandenen Images sowie die Lizenzierung elektronischer Ressourcen für die sächsischen Hochschulbibliotheken. ${ }^{8}$ Das SMWK setzte das Programm mit der avisierten Ausstattung von jährlich 2,5 Millionen Euro, die durch die SLUB verwaltet werden, in den Haushaltsverhandlungen durch; es wurde im Zuge der Verabschiedung des Doppelhaushalts 2015/2016 durch den Sächsischen Landtag und im Doppelhaushalt 2017/2018 erneut beschlossen.

Aus dem LDP werden in jedem Jahr 700000 Euro für den konsortialen Kauf elektronischer Ressourcen genutzt, über deren Verwendung in den Konsortialsitzungen konsensual befunden wird. Diese Mittel werden nicht mit einem zugewiesenen Schlüssel (z.B. nach FTE) auf die Hochschulbibliotheken verteilt, sondern nach der Entscheidung über die Lizenzierung von Produkten zentral von der SLUB als Mittelbewirtschafterin finanziert. Dieses Modell der zentralen Finanzierung sendet auch in die Politik ein starkes Signal des konsortialen Handelns. Maßgabe des Verfahrens ist die Lizenzierung elektronischer Medien für den aktuellen Forschungsbedarf. So wurden über EBA-Modelle die für die Hoch-

\footnotetext{
8 Vgl. https://www.slub-dresden.de/sammlungen/landesdigitalisierungsprogramm/. Bereits in den ersten Entwürfen des Programms bildeten die Lizenzierung bzw. der Kauf elektronischer Ressourcen eine der vorgesehenen Komponenten innerhalb des Erwerbungskonsortiums. Der Verfasser, der am Entwurf des Programms beteiligt war und die Verfahrensrichtlinie des Programms für 2015/16 schrieb, die bis auf wenige aktuelle Anpassungen auch für die laufende Periode des LDP gilt, erlebte in der Zeit der Abordnung (2014/2015) an das Sächsische Staatsministerium für Wissenschaft und Kunst (SMWK) ebenso die politischen Prozesse.
} 
schulbibliotheken bestgenutzten Titel der Verlage de Gruyter und Wiley gekauft, die die Wissenschaft in der Literaturversorgung erheblich unterstützt haben.

Zwischen den für konsortiale Zwecke der SLUB zugewiesenen Mitteln und denen des LDP besteht eine strikte Trennung, auch bei der Verteilung und Verwendung. Wenngleich eine gegenseitige Deckungsfähigkeit möglich wäre, folgt diese Separierung der Programmidee, eigene Akzente gegenüber der „normalen“ Lizenzierung zu setzen. Bei der erhofften Verlängerung des LDP über 2018 hinaus soll die Lizenzierung elektronischer Medien weiter wie bisher verankert werden, da die Ergebnisse für alle Hochschulen deutlich und erkennbar positiv sind. Wenngleich die Lizenzierung elektronischer Ressourcen durch das LDP anders als die erste Programmlinie der Retrodigitalisierung - kaum im öffentlichen Interesse steht, wirkt sie sich erheblich strukturpolitisch ordnend auf die sächsischen Hochschulbibliotheken aus - nicht zuletzt ein wichtiger Zweck des Programms. Damit ist die Programmlinie Lizenzierung in politischer Hinsicht und bei den bislang erzielten Ergebnissen ein echter Erfolg.

\section{Ein Blick in die (aktuelle) Zukunft}

Die Medienwelt im Umbruch bedeutete und bedeutet auch für das sächsische Erwerbungskonsortium neue Herausforderungen. Bereits 2014/2015 wurde die Konsortialstrategie geschärft, den neuesten Anforderungen angepasst ${ }^{9}$ und damit die Ablösung der Einzelabonnements der Hochschulbibliotheken zugunsten konsortialer Landeslizenzen entschieden vorangetrieben. Die Verträge mit den Anbietern berücksichtigten nun die Open-Access-Komponente, ebenso wie Remote Access und die Lieferung von Metadaten. Das weist in die Zukunft des Konsortiums, das sich schließlich nie als bloße Einkaufsgemeinschaft verstanden hat. Die AG Erwerbungskoordinierung definiert vielmehr bestimmte Standards, die z. B. im Metadatenmanagement bei den konsortial lizenzierten Produkten gelten. Mit dem Beitritt der SLUB zum finc-Index, den die Universitätsbibliothek Leipzig entwickelte, intensiviert sich die Zusammenarbeit der beiden Bibliotheken noch. Beide Einrichtungen befinden sich bereits jetzt in enger Abstimmung und Arbeitsteilung bei Analyse und Processing von Metadaten.

Doch werden sich die (hochschul-)politischen Rahmenbedingungen ändern. Bei einem hoffentlich positiven Ausgang der DEAL-Verhandlungen mit SpringerNature, Wiley und Elsevier besteht die Möglichkeit des Beitritts der Bi-

9 Vgl. Golsch, Landeslizenzen (wie Anm. 4). 
bliotheken $\mathrm{zu}$ diesen Konsortien. Voraussichtlich entfalten die Lizenzierungsbestimmungen der DEAL-Verträge zugleich eine gewisse Vorbildwirkung. Ob die Teilnahme daran aus zentralen Mitteln des Freistaats finanziert wird oder aus den Etats der Bibliotheken selbst, wird dann zu besprechen sein. Wie die Entscheidung auch fällt: Die Finanzierung wird sich auf die zukünftige Strategie des Konsortiums auswirken. Dass überall nationale Großlizenzen die länderweisen oder fachlich ausgerichteten Konsortien ablösen, ist für die nächsten Jahre sicher auszuschließen. Die Frage jedoch, wie sich fachliche zu regionalen Erwerbungszusammenschlüssen verhalten, dürfte noch mehr als bisher diskutiert werden. Dies muss in Sachsen zudem vor dem Hintergrund des Hochschulentwicklungsplanes 2025 betrachtet werden, der die Verlegung und Konzentration von Fächern an einzelnen Universitäten und Hochschulen vorsieht. ${ }^{10}$ Die damit verbundene Verlagerung von Fächern wird sich in den zukünftigen konsortialen Entscheidungen widerspiegeln. Nicht zuletzt hängen die Lizenzierungen von der Mittelausstattung der Hochschulbibliotheken ab, für die die seit Jahren nicht gestiegenen Etats, aber parallel dazu erhöhten Preise der Anbieter zunehmend schwieriger zu handhaben sind. Etatverschiebungen schaffen einen nur kurzfristigen und überschaubaren Ausgleich. Notwendig erscheint stattdessen eine Anpassung der entsprechenden Erwerbungsetats. Umso wichtiger ist die baldige Klarheit über die Konditionen der DEAL-Verträge, damit die Bibliotheken ihre Etats planen können. Mit den drei Gesprächspartnern der DEALVerhandlungen Springer, Wiley und Elsevier verfügt das Sachsenkonsortium über Verträge, die jeweils zum 31. Dezember 2018 auslaufen. ${ }^{11}$

Ebenso bekommen bei konsortialen Entscheidungen über den Bestandsaufbau neue Arten des Nutzungsverhaltens, neben der absoluten Nutzung und dem Verhältnis Kosten pro Download, mehr Gewicht. So befasst sich die AG Erwerbungskoordinierung seit 2017 mit dem Publikationsverhalten der Wissenschaftler der Technischen Universität Chemnitz in den Zeitschriften von SpringerNature, was sie mittelfristig auf andere Hochschulen ausdehnen und als bibliometrische Komponente in den Erwerbungsentscheidungen einkalkulieren wird. Die SLUB bereitet Ähnliches derzeit anhand der E-Journals einzelner Verlage vor. Ebenfalls 2017 prüfte die AG erstmals die Lizenzierung bzw.

10 Vgl. http://www.studieren.sachsen.de/download/HEP_2025_1.pdf.

11 Da die Haushaltsaufstellungsverfahren für den Doppelhaushalt 2019/2020 mutmaßlich vor dem Ergebnis der DEAL-Verhandlungen abgeschlossen sein müssen, können die DEAL-Ergebnisse hier wohl nicht berücksichtigt werden. Insgesamt wird dies viele Bibliotheken berühren, wobei offen ist, ob die Erwerbungsetats im großen Stile dafür herangezogen werden. Es bleibt eher zweifelhaft, dass durch die DEAL-Verträge erhebliche Einsparungen erfolgen; der Erfolg abgeschlossener DEAL-Verträge bemisst sich vermutlich besser an der Versorgung mit der wissenschaftlichen Literatur insgesamt, dem Open Access $u$. a. 
den „Freikauf“ von Medien zur Umstellung auf Open Access, nahm diese jedoch aufgrund des inhaltlichen Angebots und der damit noch zu geringen Spezifik für die größtenteils technisch-naturwissenschaftlichen Fächer der sächsischen Hochschulen nicht in die Auswahl. Bei diesem Modell geht der Bestandsaufbau ebenfalls neue Wege und die klassische Nutzungsmessung als Begründung für Weiterlizenzierungen ist damit obsolet. Gleichzeitig würdigt das Konsortium einen nicht zuletzt politischen Aspekt und unterstützt Open Access, was jedoch genau wie alle anderen Erwerbungen wirtschaftlich begründet sein muss. Auch hier werden die Anbieter, wie bereits die Verlage, nicht umhinkommen, hinreichend attraktive und spezifische E-Book- und E-Journalpakete zu schnüren, die wissenschaftsrelevant sind und nicht nur aus Unterstützungsmotiven erworben werden.

Bei zukünftigen Verträgen muss konsortial darauf geachtet werden, inwieweit diese Finanzierung als Geschäftsaufwand abgebildet wird. ${ }^{12}$ Weiterhin wird beobachtet, wie sich die Angebote bei Lizenz- und Abonnementverwaltungen der entsprechenden Dienstleister angesichts der drohenden Verluste durch nationale DEAL-Verträge entwickeln und wie diese konsortial genutzt werden können. Bei einer stärkeren Verankerung von Open-Access-Publikationsgebühren in konsortialen Erwerbungsentscheidungen bedeutet die Einzelabrechnung von APCs einen erheblichen Aufwand. Auch hier sind die Konsortien gefragt, wirtschaftlich und organisatorisch tragfähige Modelle mit den Anbietern zu gestalten.

Über gute und geeignete Erwerbungsmodelle hinaus sollen technisch-organisatorisch einheitliche Rahmenbedingungen geschaffen werden. Mit dem von der UB Leipzig entwickelten Amsl-ERM (Electronic Resource Management) wird bereits jetzt für konsortiale Belange ein gemeinsames Werkzeug genutzt, z. B. bei der Speicherung und Auswertung von Nutzungsdaten oder bei der Lizenzverwaltung allgemein. Das Format und weitere technische Standards der Metadaten für Artikel gekaufter E-Journal-Pakete werden momentan vertraglich festgelegt. Auch hier soll, wie das Konsortium derzeit mit einzelnen Anbietern und Verlagen verhandelt, eine größere Einheitlichkeit zugunsten der Teilnehmerbibliotheken erzielt werden. Die Frage der gesonderten Vergütung von zu liefernden Metadaten wird abhängig vom Umfang der einzelnen Daten beantwortet. Umfassen sie besondere Angaben, die sonst nicht $\mathrm{zu}$ beziehen sind und

12 Das Hinzuziehen eines externen Dienstleisters ist durchaus möglich, um das Fehlen einer konsortialen Geschäftsstelle in diesem Bereich zumindest teilweise abzufangen. Die Leitung und Finanzverwaltung des Sachsenkonsortiums decken derzeit der Konsortialführer und dessen Sekretariat sowie zwei weitere Kolleginnen der Abteilung Bestandsentwicklung und Metadaten arbeitsteilig ab. 
hinter denen ein zu spezifizierender Aufwand steht, ist eine Vergütung möglich, wenngleich das grundsätzliche Ziel in der Verhandlungsführung die selbstverständliche Integration der Metadaten in den Gesamtpreis ist.

Nicht zu vergessen sind die Anforderungen an ein zukünftiges Bibliotheksmanagementsystem. Die UB Leipzig hat 2016, die SLUB Dresden 2017 Libero 6 von LibIT implementiert, das mittelfristig durch eine neue Version oder durch ein neues Produkt abgelöst wird. Schon jetzt wird erörtert, für welche Bereiche welcher Bedarf bei einem Bibliotheksmanagementsystem besteht. Auch im Konsortium wird dies debattiert werden. Ebenso besteht beim Digital Rights Management (DRM) konsortialer Handlungsbedarf. Wie bei anderen Konsortien ist das DRM in allen Verhandlungen der AG ein wichtiges Thema der Diskussionen, die aber noch nicht immer zu einem befriedigenden Ergebnis führen. Zukünftig können neue Verträge etwa Datamining $u$. a. gesondert berücksichtigen, was das neue Urheberrechts-Wissensgesellschafts-Gesetz erleichtert.

Intensivieren wird sich auch die Zusammenarbeit mit dem Konsortium der baden-württembergischen Bibliotheken. Beide Konsortien nehmen an den Sitzungen des jeweils anderen Konsortiums teil. Weiterhin besteht mit thüringischen Hochschulbibliotheken bei einigen Produkten eine enge Partnerschaft. Über diese Kooperation hinaus wird die verstärkte Abstimmung mit anderen Länder- und Fachkonsortien angestrebt. Ob das Konzept des sächsischen Konsortiums, zumindest mit einem Produkt über die Landesgrenzen hinaus konsortiale Lizenzen vorzuhalten, ausgebaut wird, hängt von den Rahmenbedingungen und letzten Endes der Finanzierung der entsprechenden IT- und Personaldienstleistungen ab. Jedoch sind für diese strategischen Entscheidungen auch zukünftig der Ausbau von Partnerschaften und der Aspekt der Metadatenexpertise wichtig und es handelt sich mitnichten um bloße fiskalische Entscheidungen.

Die Konsortialführung hat die Aufgabe, diese zahlreichen Anforderungen an das Konsortium zu bündeln, gemeinsam mit den Teilnehmern zu bewerten und Lösungen zu entwickeln. Die unterschiedlichen Interessen und Anforderungen zusammenzuführen, liegt allerdings nicht nur in der Verantwortung der Konsortialführung, sondern alle Teilnehmer können daran partizipieren. Werden Angebote nicht durch das ganze Konsortium lizenziert, besteht etwa die Möglichkeit, kleinere Konsortien innerhalb der AG Erwerbungskoordinierung zu bilden, um damit ebenfalls Preis- und Servicevorteile zu erlangen. Somit verwaltet die Arbeitsgemeinschaft mehr als die „großen“ Lizenzen, die das gesamte Konsortium bezieht.

Auch künftig setzt die AG Erwerbungskoordinierung auf die bewährte Arbeitsteilung und beteiligt die Bibliotheken des Konsortiums an allen Prozessen verantwortlich. Die SLUB als Konsortialführerin steuert also ein wichtiges 
Gremium der Bibliotheks- und wissenschaftlichen Informationsinfrastruktur in Sachsen, das seit mittlerweile 24 Jahren nachhaltig wirkt und sich auch in $\mathrm{Zu}$ kunft den Rahmenbedingungen und neuen Entwicklungen nicht nur anpasst, sondern diese aktiv mitgestaltet. Dass der eingeschlagene Weg der Partnerschaften und strategischen Abstimmungen richtig war und ist, beweist sich dabei jeden Tag. 
